\title{
Universality in hadronic and nuclear collisions at high energy
}

\author{
P. Castorina $\odot,{ }^{1,2}$ A. Iorio $\odot,^{2}$ D. Lanteri $\odot,{ }^{1,3}$ H. Satz $\odot,{ }^{4}$ and M. Spousta $\odot^{2}$ \\ ${ }^{1}$ INFN, Sezione di Catania, I-95123 Catania, Italy \\ ${ }^{2}$ Institute of Particle and Nuclear Physics, Faculty of Mathematics and Physics, Charles University, V Holešovičkách 2, \\ 18000 Prague 8, Czech Republic \\ ${ }^{3}$ Dipartimento di Fisica e Astronomia, Università di Catania, I-95123 Catania, Italy \\ ${ }^{4}$ Fakultät für Physik, Universität Bielefeld, D-33501 Bielefeld, Germany
}

(Received 26 December 2019; revised manuscript received 28 February 2020; accepted 27 April 2020; published 11 May 2020)

\begin{abstract}
Recent experimental results in proton-proton and in proton-nucleus collisions at Large Hadron Collider energies show a strong similarity to those observed in nucleus-nucleus collisions, where the formation of a quark-gluon plasma is expected. We discuss the comparison between small colliding systems and nucleusnucleus collisions, for (a) the strangeness suppression factor $\gamma_{s}$ and yields of multi-strange hadrons; (b) the average transverse momentum, $p_{t}$, with particular attention to the low $p_{t}$ region where soft, nonperturbative effects are important; and (c) the elliptic flow scaled by the participant eccentricity. The universal behavior in hadronic and nuclear high energy collisions emerges for all these observables in terms of a specific dynamical variable which corresponds to the entropy density of initial system in the collision and which takes into account the transverse size of the initial configuration and its fluctuations.
\end{abstract}

DOI: 10.1103/PhysRevC.101.054902

\section{INTRODUCTION}

Recent experimental results in proton-proton $(p p)$ and proton-nucleus $(p A)$ collisions at the Large Hadron Collider (LHC) and Relativistic Heavy Ion Collider (RHIC) show a strong similarity to those observed in nucleus-nucleus $(A A)$ collisions, where the formation of a quark-gluon plasma is expected. Many different signatures [1-9] support the conclusion that the system created in high energy, high multiplicity collisions with "small" initial settings, i.e., $p p$ and $p A$, is essentially the same as that one produced with "large" initial $A A$ configurations.

The ALICE Collaboration reported [1] the enhanced production of multistrange hadrons, previously observed in $\mathrm{PbPb}$ collisions [10], in high energy, high multiplicity, $p p$ events. The strangeness enhancement was suggested to be present in high-multiplicity $p p$ collisions on theoretical grounds in Refs. [11,12] by considering a specific dynamical variable corresponding to the initial entropy density of the collisions, which takes into account the transverse size (and its fluctuations) of the initial configuration in high multiplicity events $[13,14]$. Noticeably, the energy loss in $A A$ collisions was also shown to scale in the same dynamical variable [15].

Published by the American Physical Society under the terms of the Creative Commons Attribution 4.0 International license. Further distribution of this work must maintain attribution to the author $(s)$ and the published article's title, journal citation, and DOI. Funded by $S C O A P^{3}$.
An important similarity between $p p, p A$, and $A A$ collisions was identified also in several measurements of long-range dihadron azimuthal correlations $[3,4,6,16]$ indicating universally present flowlike patterns.

The similarity of the average transverse momentum $\left(p_{t}\right)$ between $p p, p A$, and $A A$ collisions was discussed in Refs. [17-19], where the scaling of $p_{t}$ as a function of the variable $N_{\text {track }} / A_{T}$ ( $N_{\text {track }}$ being the multiplicity and $A_{T}$ the transverse area of the initial system) was explored in the framework of color glass condensate (CGC), where also the geometrical scaling of direct-photon production in hadron collisions at RHIC and LHC energies has been obtained in terms of the saturation scale, proportional to the transverse entropy density [20].

The previously discussed similarities indicate that a few dynamical ingredients, common to the different initial settings, drive the particle production, independently of the complexity of the nonequilibrium dynamics with annihilation and creation of many interacting quarks and gluons and hadronization of final partons.

In this paper, we discuss some pieces of the mosaic of this universal behavior in a unified way. More precisely, for small colliding systems versus $A A$ collisions, we compare (a) the strangeness enhancement, refining previous analyses $[13,14]$; (b) the mean $p_{t}$, with particular attention to the low $p_{t}$ region where the soft, nonperturbative effects are important; and (c) the elliptic flow, $v_{2}$, where a scaling behavior has been already observed [21-23] for different nuclei $(\mathrm{Au}, \mathrm{Cu}$, and $\mathrm{Pb}$ ) by considering the ratio $v_{2} / \epsilon_{\text {part }}$, where $\epsilon_{\text {part }}$ is the participant eccentricity, defined for example in [23-26]. All the comparisons speak in favor of the universal, initial entropy 
density driven mechanism for the particle production across different colliding systems.

\section{EMERGENT UNIVERSALITY}

Let us recall that the initial entropy density $s_{0}$ is given in the one-dimensional hydrodynamic formulation [27] by the form

$$
s_{0} \tau_{0} \simeq \frac{1.5}{A_{T}} \frac{d N_{c h}^{x}}{d y}=\left.\frac{1.5}{A_{T}} \frac{N_{\mathrm{part}}^{x}}{2} \frac{d N_{c h}^{x}}{d y}\right|_{y=0},
$$

with $x \simeq p p, p A, A A$. Here $A_{T}$ is the transverse area, $\left(d N_{c h}^{x} / d y\right)_{y=0}$ denotes the number of produced charged secondaries, normalized to half the number of participants $N_{\text {part }}^{x}$, in reaction $x$, and $\tau_{0}$ is the formation time. The initial entropy density is directly related to the number of partons per unit of transverse area and, due to the large fluctuations in high multiplicity events, one needs a reliable evaluation of the transverse area for different collisions.

In studying the strangeness enhancement and the average $p_{t}$, we use results from Glauber Monte Carlo (MC) [28] to obtain $A_{T}$ as a function of multiplicity for $A A$ and for $p \mathrm{~Pb}$ collisions. For $p p$ collisions the effective transverse area is sensitive to the fluctuations of the gluon field configurations and therefore we apply the CGC parametrization of the transverse size as a function of multiplicity [17-19].

On the other hand, for the scaling behavior of the elliptic flow, namely of the ratio $v_{2} / \epsilon_{\text {part }}$, the effective transverse area, $S$, of the initial setting is the one related to $\epsilon_{\text {part }}$. The $S$ is evaluated by MC simulations in Refs. $[22,26]$ for $A A$ and in Ref. [29] for $p p$ collisions.

\section{UNIVERSALITY IN STRANGENESS PRODUCTION}

In Refs. [13,14] the parameter $\gamma_{s} \leqslant 1$, which describes the strangeness suppression in the statistical hadronization model (SHM) [30], was studied as a function of the variable from Eq. (1), by using an approximate evaluation of the transverse area for $p p, p \mathrm{~Pb}$, and $A A$ collisions.

Here we use an improved evaluation of the transverse area for $A A, p \mathrm{~Pb}$, and $p p$ collisions as described in the previous section. The resulting scaling behavior for the strangeness production is reported in Fig. 1, where $\gamma_{s}$ for $A A$ at different energies and centralities are shown along with those for $p \mathrm{~Pb}$ and $p p$ collisions. The data refer to $p p$ at energy $\sqrt{s}=26 \mathrm{GeV}$ to $7 \mathrm{TeV}[31,32]$, to $p \mathrm{~Pb}$ at $\sqrt{s}=2.76 \mathrm{TeV}$ [11-14,33], to $\mathrm{PbPb}$ at $\sqrt{s}=2.76 \mathrm{TeV}$ [34], to AuAu at $\sqrt{s}=$ $19.6,27,39$ and $200 \mathrm{GeV}$ [35], and to $\mathrm{CuCu}$ at $\sqrt{s}=200 \mathrm{GeV}$ [32].

The universal trend shows that $\gamma_{s}$ increases with the parton density in the transverse plane, up to the fixed point $\gamma_{s}=1$, where any suppression disappears.

The result in Fig. 1 has been obtained by estimating $\gamma_{s}$ in a specific SHM $[31,32,34,35]$. On the other hand, different versions of the model, which take into account various dynamical aspects of the hadron resonance gas in thermal equilibrium (e.g., set of included resonances or excluded volume) $[37,38]$ could give slightly different values for $\gamma_{s}$. While the impact of this can be further tested using different SHM, we checked the

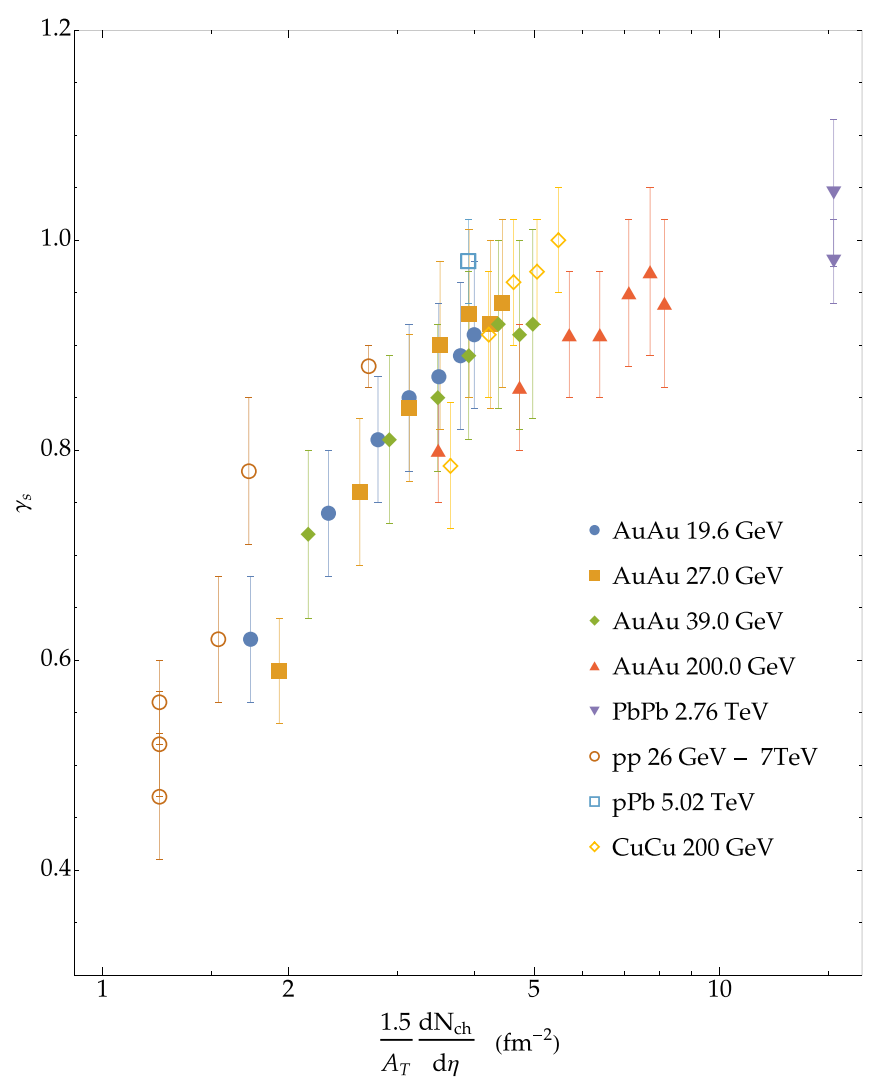

FIG. 1. The strangeness suppression factor $\gamma_{s}$ as a function of initial entropy density evaluated for data from Refs. [31,32,34,35]. The Phobos parametrization [36] for the relation between charge multiplicity, energy and the number of participants is applied for RHIC data.

universality by a model independent analysis. In Fig. 2, ratios of yields of $K, \Lambda, \Xi$, and $\Omega$ hadrons to pions were evaluated as a function of initial entropy density for $\mathrm{PbPb}[10,39-41]$, $p \mathrm{~Pb}[2,42]$, and $p p[1,43]$ data. The production of particles containing strangeness exhibits universality for all the tested collision systems.

Figures 1 and 2 represent two different aspects of universality, since the first one presents a large range of collision energy, whereas the second one, at fixed LHC energy, is a more genuine indication of the role of the system size.

\section{UNIVERSALITY IN MEAN TRANSVERSE MOMENTUM}

As recalled, the scaling of the average $p_{t}$ as a function of the variable $N_{\text {track }} / A_{T}$ has been discussed in [17-19]. Here we analyze the average $p_{t}$ in the low transverse momentum region where the soft, nonperturbative effects in the particle production are more important due to running of the strong coupling constant than in the higher $p_{t}$ range. The behavior of the average $p_{t}$ is evaluated in the region $0.15<p_{t}<1.5 \mathrm{GeV}$ for different colliding systems as a function of the dynamical variable from Eq. (1). The results are shown in Fig. 3 for the data from Refs. [44-47]. One can see that the average $p_{t}$ for soft particle production follows the same slowly increasing 


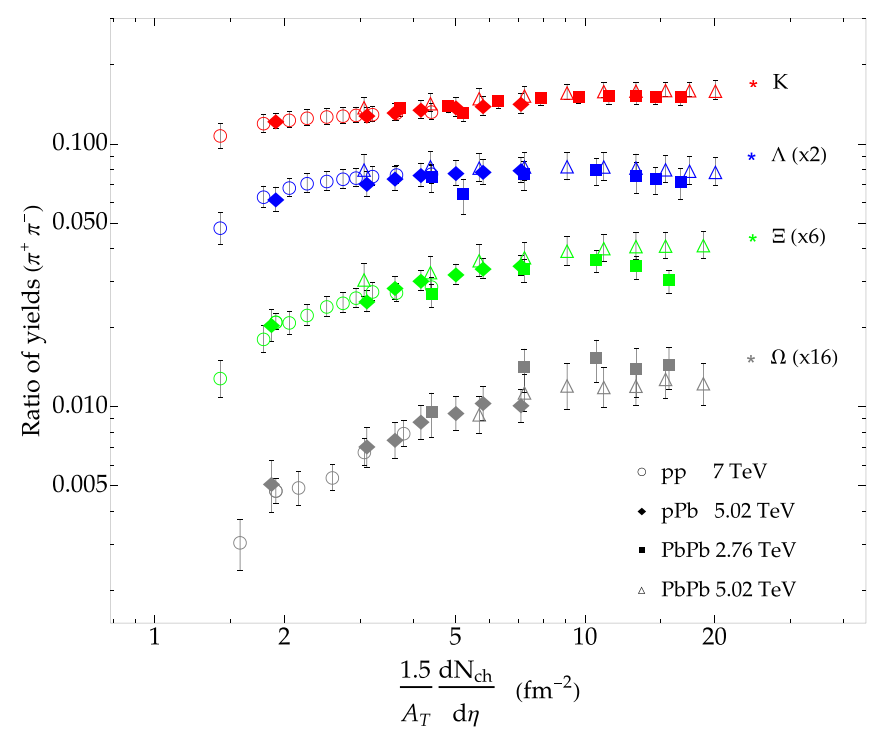

FIG. 2. The strangeness production quantified in terms of the ratio of yields of $K, \Lambda, \Xi$, and $\Omega$ hadrons to pions evaluated as a function of initial entropy density for data from Refs. [1,2,10,39-43].

trend for all the collisional systems. Discussion of a possible scaling of the hard component has been carried out, e.g., in Ref. [48].

\section{UNIVERSALITY IN THE ELLIPTIC FLOW}

In noncentral collisions, the beam direction and the impact parameter vector define a reaction plane for each event. If the nucleon density within the nuclei is continuous, the initial

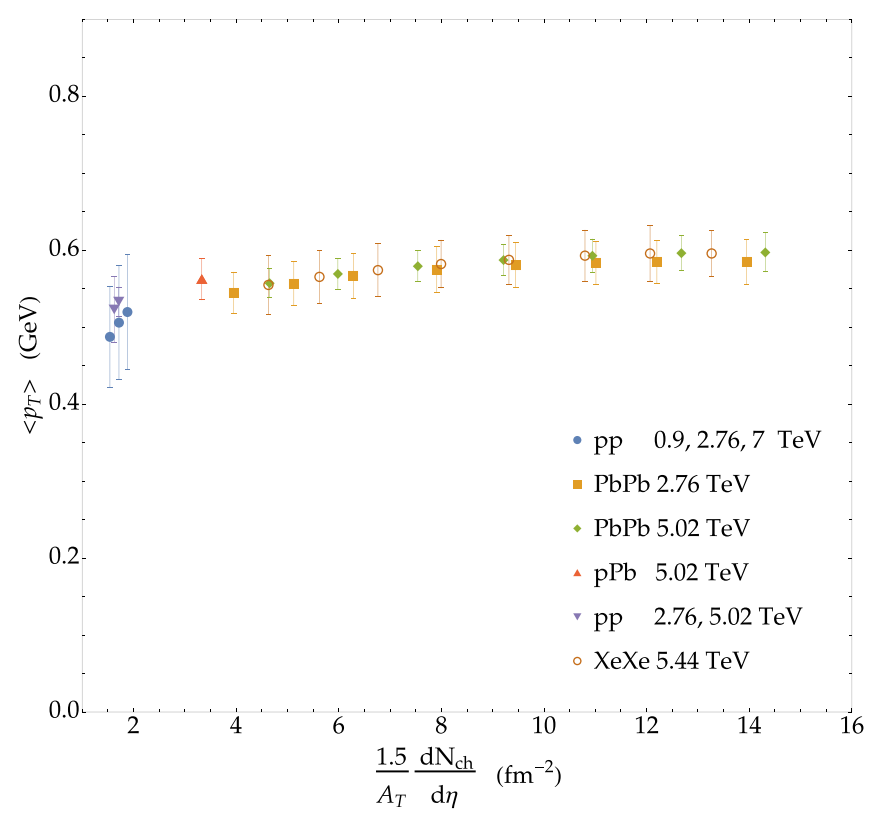

FIG. 3. Average $p_{t}$ as a function of initial entropy density evaluated in the interval of $0.15<p_{t}<1.5 \mathrm{GeV}$ for the data from Refs. [44-47].

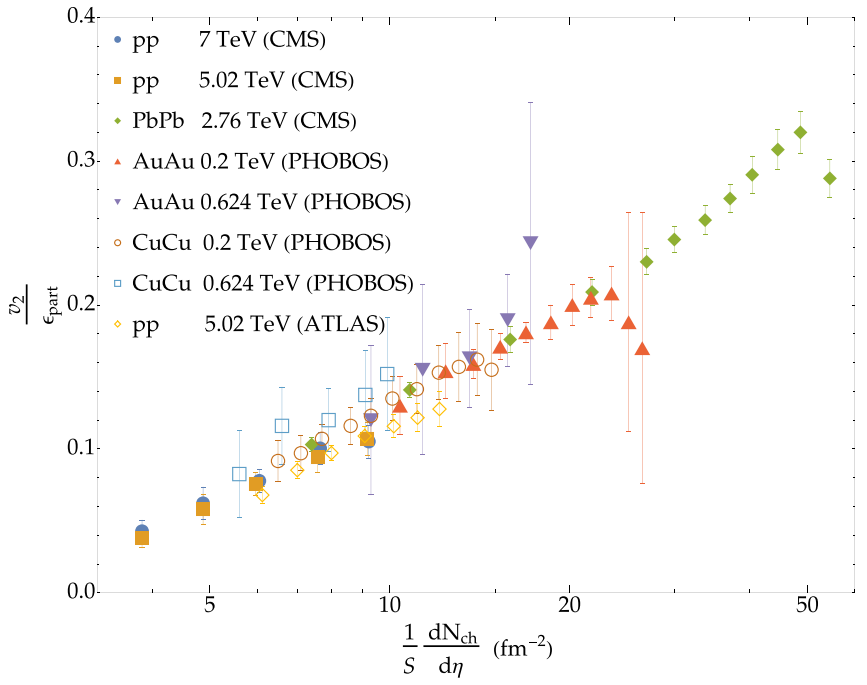

FIG. 4. The $v_{2} / \epsilon_{\text {part }}$ values for $p p, \mathrm{PbPb}, \mathrm{AuAu}$, and $\mathrm{CuCu}$ evaluated as a function of entropy density for data from Refs. [3,21,22,50].

nuclear overlap region has an "almondlike" shape and the impact parameter determines uniquely the initial geometry of the collision.

In a more realistic description, where the position of the individual nucleons that participate in inelastic interactions is considered, the overlap region has a more irregular shape and the event-by-event orientation of the almond fluctuates around the reaction plane $[25,49]$. Therefore, in the analysis of the elliptic flow where the fluctuations are important, the geometrical eccentricity is replaced by the participant eccentricity, $\epsilon_{\text {part }}$, defined using the actual distribution of participants. The size of the fluctuation in $\epsilon_{\text {part }}$ and its correlated transverse area $S$ (different from the geometrical one) are evaluated by Glauber MC as previously described.

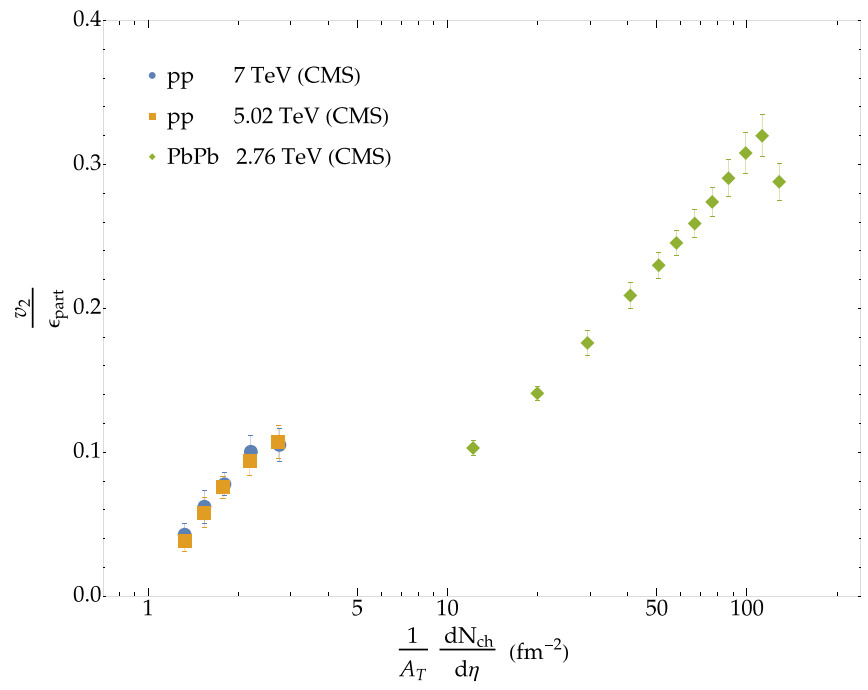

FIG. 5. The $v_{2} / \epsilon_{\text {part }}$ values for $p p$ and $\mathrm{PbPb}$ evaluated as a function of entropy density when the geometrical transverse area $A_{T}$, rather than $S$, is used in the evaluation of the initial energy density for data 
The scaling of $v_{2} / \epsilon_{\text {part }}$ versus the initial entropy density is depicted in Fig. 4 for $A A[21,22]$ and $p p[3,50]$. One can see that the $p p$ trend, at lower values, is smoothly followed by the data points from $A A$ collisions.

To clarify the different role of $A_{T}$ versus $S$, Fig. 5 shows that the scaling in $v_{2} / \epsilon_{\text {part }}$ is not observed if one considers $A_{T}$ rather than $S$ in evaluating the initial entropy density.

\section{HOW TO CHECK THE UNIVERSAL TREND}

The analyses of $\gamma_{s}$, average $p_{T}$, and $v_{2} / \epsilon_{\text {part }}$ presented above support the conclusion that at fixed entropy density the "coarse-grain" features of the quark-gluon system formed in high energy collisions are independent of the initial configuration. The scaling variable [Eq. (1)] is a function of multiplicity and the transverse area, and one can evaluate at which multiplicity one can expect the same behavior in highmultiplicity $p p$ and $\mathrm{PbPb}$ collisions, by solving the equation $(d N / d \eta)_{A A} / A_{T}^{A A}=x / A_{T}^{p p}(x)$ for $x$, which is the multiplicity in $p p$. The result is shown in Table $\mathrm{I}$ for $\mathrm{PbPb}$ collisions at 5.02 $\mathrm{TeV}$ which represent the largest available heavy-ion dataset at the LHC. The values from Table I can be used in subsequent experimental or phenomenological studies aiming to further check the universal trends in hadronic and nuclear collisions using high-multiplicity $p p$ collisions at the largest available LHC energies.

\section{COMMENTS AND CONCLUSIONS}

High energy and high multiplicity events produced in small colliding systems show dynamical behavior very similar to
TABLE I. $d N_{c h} / d \eta$ in $\mathrm{PbPb}$ at $5.02 \mathrm{TeV}$ and $p p$ for different values of the variable in Eq. (1).

\begin{tabular}{lccc}
\hline \hline$\frac{1.5}{A_{T}} \frac{d N_{c h}}{d \eta}$ & $\left(\frac{d N_{c h}}{d \eta}\right)_{p p}$ & $\left(\frac{d N_{c h}}{d \eta}\right)_{\mathrm{PbPb}}$ & $\mathrm{PbPb}$ centrality \\
\hline $20.1 \pm 0.8$ & $100 \pm 4$ & $1943 \pm 56$ & $0-5 \%$ \\
$17.5 \pm 1.1$ & $87 \pm 5$ & $1587 \pm 47$ & $5-10 \%$ \\
$15.4 \pm 0.9$ & $76 \pm 4$ & $1180 \pm 31$ & $10-20 \%$ \\
$12.2 \pm 0.6$ & $60.6 \pm 3.1$ & $649 \pm 13$ & $20-40 \%$ \\
$8.3 \pm 0.7$ & $41.2 \pm 3.4$ & $251 \pm 7$ & $40-60 \%$ \\
$5.2 \pm 0.8$ & $26 \pm 4$ & $70.6 \pm 3.4$ & $60-80 \%$ \\
$3.1 \pm 1.1$ & $12.4 \pm 3.0$ & $17.5 \pm 1.8$ & $80-90 \%$ \\
\hline \hline
\end{tabular}

that present in $A A$ collisions. Observations made in this paper suggest that the dynamical behavior is largely driven by the initial entropy density, that is by the parton density in the transverse plane. A clear quantification of limits on the presence of jet quenching in small colliding systems (see, e.g., discussions and new measurements in Refs. [51-53]) or more detailed correlation measurements (see, e.g., recent work in Refs. [54-56]) may help to improve understanding of this similarity. This kind of measurements can be done in detail at the LHC or RHIC or at a $100 \mathrm{TeV} p p$ collider which is considered for the future [57] and which would significantly enhance the reach of multiplicities in $p p$ collisions.

\section{ACKNOWLEDGMENTS}

A.I. and M.S. are partially supported by Univerzita Karlova v Praze (Grant No. UNCE/SCI/013), and M.S. is also supported by Grant Agency of the Czech Republic (Grant No. GAČR 18-12859Y).
[1] J. Adam et al. (ALICE Collaboration), Nat. Phys. 13, 535 (2017).

[2] B. Abelev et al. (ALICE Collaboration), Phys. Lett. B 728, 25 (2014).

[3] V. Khachatryan et al. (CMS Collaboration), Phys. Lett. B 765, 193 (2017).

[4] G. Aad et al. (ATLAS Collaboration), Phys. Rev. Lett. 116, 172301 (2016)

[5] V. Khachatryan et al. (CMS Collaboration), J. High Energy Phys. 09 (2010) 091.

[6] V. Khachatryan et al. (CMS Collaboration), Phys. Rev. Lett. 116, 172302 (2016).

[7] C. Aidala et al. (PHENIX Collaboration), Nat. Phys. 15, 214 (2019).

[8] E. K. G. Sarkisyan, A. N. Mishra, R. Sahoo, and A. S. Sakharov, Phys. Rev. D 94, 011501(R) (2016).

[9] C. Loizides, Nucl. Phys. A 956, 200 (2016).

[10] B. Abelev et al. (ALICE Collaboration), Phys. Lett. B 728, 216 (2014); 734, 409 (2014).

[11] P. Castorina and H. Satz, Eur. Phys. J. A 52, 200 (2016).

[12] P. Castorina, S. Plumari, and H. Satz, Int. J. Mod. Phys. E 25, 1650058 (2016).

[13] P. Castorina, S. Plumari, and H. Satz, Int. J. Mod. Phys. E 26, 1750081 (2017).
[14] P. Castorina, S. Plumari, and H. Satz, Int. J. Mod. Phys. E 27, 1891001 (2018).

[15] A. Adare et al. (PHENIX Collaboration), Phys. Rev. C 93, 024911 (2016).

[16] G. Aad et al. (ATLAS Collaboration), Phys. Rev. Lett. 110, 182302 (2013).

[17] L. McLerran, M. Praszalowicz, and B. Schenke, Nucl. Phys. A 916, 210 (2013)

[18] A. Bzdak, B. Schenke, P. Tribedy, and R. Venugopalan, Phys. Rev. C 87, 064906 (2013).

[19] L. McLerran and P. Tribedy, Nucl. Phys. A 945, 216 (2016).

[20] C. Klein-Boesing and L. McLerran, Phys. Lett. B 734, 282 (2014).

[21] R. Nouicer et al. (PHOBOS Collaboration), J. Phys. G 34, S887 (2007).

[22] S. Chatrchyan et al. (CMS Collaboration), Phys. Rev. C 87, 014902 (2013).

[23] S. Manly et al. (PHOBOS Collaboration), Nucl. Phys. A 774, 523 (2006).

[24] R. S. Bhalerao and J.-Y. Ollitrault, Phys. Lett. B 641, 260 (2006).

[25] B. Alver et al. (PHOBOS Collaboration), Phys. Rev. C 77, 014906 (2008).

[26] T. Hirano and Y. Nara, Phys. Rev. C, 79, 064904 (2009). 
[27] J. D. Bjorken, in Current Induced Reactions, International Summer Institute on Theoretical Particle Physics in Hamburg 1975, edited by J. G. Körner, G. Kramer, and D. Schildknecht, Lecture Notes in Physics Vol. 56 (Springer, Berlin, 1976), p. 93.

[28] C. Loizides, J. Kamin, and D. d'Enterria, $\begin{array}{llllll}\text { Phys. Rev. C 97, } 054910 & \text { (2018); 99, } 019901\end{array}$ (2019).

[29] E. Avsar, C. Flensburg, Y. Hatta, J.-Y. Ollitrault, and T. Ueda, Phys. Lett. B 702, 394 (2011).

[30] F. Becattini, J. Phys.: Conf. Ser. 5, 175 (2005).

[31] F. Becattini, in 33rd Workshop of the INFN Eloisatron Project, Erice, Italy, October 19-25, 1996 (unpublished), pages 74-104.

[32] J. Takahashi and R. Derradi de Souza, in 24th Winter Workshop on Nuclear Dynamics (WWND 2008), South Padre Island, Texas, April 5-12, 2008 (unpublished).

[33] P. Castorina, M. Floris, S. Plumari, and H. Satz, PoS, EPSHEP2017, 158, (2017).

[34] F. Becattini, M. Bleicher, T. Kollegger, T. Schuster, J. Steinheimer, and R. Stock, Phys. Rev. Lett. 111, 082302 (2013).

[35] L. Adamczyk et al. (STAR Collaboration), Phys. Rev. C 96, 044904 (2017).

[36] B. Alver et al. (PHOBOS Collaboration), Phys. Rev. C 83, 024913 (2011).

[37] S. Wheaton, J. Cleymans, and M. Hauer, Comput. Phys. Commun. 180, 84 (2009).

[38] A. Andronic, P. Braun-Munzinger, K. Redlich, and J. Stachel, Nature (London) 561, 321 (2018).

[39] B. Abelev et al. (ALICE Collaboration), Phys. Rev. C 88, 044910 (2013).

[40] B. Abelev et al. (ALICE Collaboration), Phys. Rev. Lett. 111, 222301 (2013).
[41] P. Kalinak, PoS EPS-HEP2017, 168 (2017).

[42] J. Adam et al. (ALICE Collaboration), Phys. Lett. B 758, 389 (2016).

[43] S. Acharya et al. (ALICE Collaboration), Phys. Rev. C 101, 044907 (2020).

[44] B. Abelev et al. (ALICE Collaboration), Phys. Lett. B 720, 52 (2013).

[45] B. Abelev et al. (ALICE Collaboration), Eur. Phys. J. C 73, 2662 (2013).

[46] S. Acharya et al. (ALICE Collaboration), J. High Energy Phys. 11 (2018) 013.

[47] S. Acharya et al. (ALICE Collaboration), Phys. Lett. B 788, 166 (2019).

[48] A. Mistra, A. Ortiz, and G. Paic, Phys. Rev. C 99, 034911 (2019).

[49] B. Alver et al. (PHOBOS Collaboration), Phys. Rev. Lett. 98, 242302 (2007).

[50] M. Aaboud et al. (ATLAS Collaboration), Phys. Rev. C 96, 024908 (2017).

[51] M. L. Mangano and B. Nachman, Eur. Phys. J. C 78, 343 (2018).

[52] B. G. Zakharov, J. Phys. G 41, 075008 (2014).

[53] G. Aad et al. (ATLAS Collaboration), Eur. Phys. J. C 80, 73 (2020).

[54] S. Acharya et al. (ALICE Collaboration), Phys. Rev. Lett. 123, 142301 (2019).

[55] A. M. Sirunyan et al. (CMS Collaboration), Phys. Rev. C 98, 044902 (2018).

[56] G. Aad et al. (ATLAS Collaboration), Eur. Phys. J. C 79, 985 (2019).

[57] N. Arkani-Hamed, T. Han, M. Mangano, and L.-T. Wang, Phys. Rep. 6521 (2016). 